$\xi=$ 国

\title{
Dufour-Soret Effects on 3D Convective Viscoelastic Fluid Flow Upon a Stretched Surface
}

\author{
M. Bhuuvaneswari ${ }^{1}$, S. Sivasankaran ${ }^{1 *}$, S. Karthikeyan², S. Rajan² \\ ${ }^{I}$ Department of Mathematics, King Abdulaziz University, Jeddah, Saudi Arabia \\ ${ }^{2}$ Department of Mathematics, Erode Arts and Science College, Erode, Tamilnadu, India \\ *Corresponding author E-mail:sd.siva@yahoo.com
}

\begin{abstract}
The purpose of this analytical work is to investigate the Dufour-Soret effects on three dimensional unsteady boundary layer flows, mass and heat transfer of a viscoelastic fluid upon a stretched surface in the existence of internal heat generation/absorption. The equations governing the flow are converted using similarity variables into a set of non-linear ordinary differential equations. The series solution is obtained by homotopy analysis. The results are analyzed for the influences of the various pertinent constants involving in the study. The mass and heat transfer rates are calculated by the localized Sherwood and Nusselt numbers along the surface.
\end{abstract}

Keywords: Mass and heat transfer; Homotopy analysis method; Dufour-Soret effects; Viscoelastic fluid.

\section{Introduction}

Boundary layer investigation of the flow upon a stretched surface has been an active area for the researchers in recent years due to the plenty of applications in engineering industries. These types of flows have appeared widely in continuous casting, hot rolling, fiber spinning, glass blowing, rolling and manufacturing plastic films. The pioneering work by Sakiadis [1] analyzed the characteristics of the flow upon the boundary layer of a stretched surface. Later, Crane [2] made an extension to this idea for the problem of two dimensional flows upon a stretching sheet. Wang [3] focused attention on steady incompressible three-dimensional viscous fluid flow due to a stretched flat surface. It appears that he was the first to derive an accurate similarity solution for the Navier-Stokes equations. The influence of radiation in steady combined convective hydro magnetic viscous incompressible fluid flow upon exponentially stretched sheet was analyzed by El-Aziz and Nabil [4]. Eswaramoorthi et al. [5] examined the consequences due to radiation in hydrodynamic convection of viscoelastic liquid upon a stretched area. Bhuvaneswari et al. [6] probed the influence of radiation together with generation of heat on convection flow upon an inclined surface surrounded by a porous material by applying the theory of Lie groups.

Even though Dufour-Soret effects seldom exist, they are relevant in areas such as geosciences or hydrology. There has always been a renewed interest in incorporating Dufour-Soret effects in convective processes in clear liquids as well as porous materials. Combined transfer of mass and heat by natural convection in a stagnating point flow of a liquid saturated porous material, incorporating the model of Darcy-Boussinesq, having blowing or suction, Dufour-Soret effects were deliberately analyzed by Postelnicu [7]. The influences of flow, mass-heat transfer on hydro magnetic chemically reacting flow in a two-dimensional irregular channel under radiation and Dufour effect was researched by Ruchi Kumar and Sivaraj [8]. The hydro magnetic combined con- vection stagnating liquid approaching an upright plate surrounded by a strongly porous space with mass and heat transport in the influence of radiation, heat generation, Dufour-Soret effects was investigated by Karthikeyan et al [9].

The homotopy analysis method (HAM), suggested by Liao [10] is one of the most efficient methods to construct approximate solutions of differential equations which are highly nonlinear. This solution method has been adapted to a variety of nonlinear differential equations. In this paper, we analyze the Dufour-Soret effects on $3 \mathrm{D}$ boundary layer heat and mass transfer upon a stretched expanse in the presence of chemical reaction, radiation and heat generation. The series solution is got by homotopy analysis method.

\section{Mathematical Formulation}

An unsteady incompressible 3D laminar flow of a viscoelastic fluid upon a stretched surface is examined. $x, y$-axes has been fixed along the stretched plane and the $z$-axis is chosen in perpendicular direction. The boundary is maintained with fixed temperature $T_{w}$ and fixed concentration $C_{w}$. These are higher than the surrounding fluid temperature $T_{\infty}$ and concentration $C_{\infty}$. The influences of radiation, chemical reaction and internal heat absorption/generation and Dufour-Soret effects are considered. Generation or consumption of the diffusing species due to the homogeneous chemical reaction is considered. The equations depicting this model are given by

$$
\begin{aligned}
& u_{x}+v_{y}+w_{z}=0 \\
& u_{t}+u u_{x}+v u_{y}+w u_{z}=v u_{z z}-k_{0}\left(u_{z z t}+u u_{x z z}+w u_{z z z}\right. \\
& \left.-u_{x} u_{z z}-u_{z} w_{z z}-2 u_{z} u_{x z}-2 w_{z} u_{z z}\right)
\end{aligned}
$$




$$
\begin{aligned}
& v_{t}+u v_{x}+v v_{y}+w v_{z}=w v_{z z}-k_{0}\left(v_{z z t}+v v_{x z z}+w v_{z z z}\right. \\
& \left.-v_{x} v_{z z}-v_{z} w_{z z}-2 v_{z} v_{y z}-2 w_{z} v_{z z}\right) \\
& T_{t}+u T_{x}+v T_{y}+w T_{z}=\alpha T_{z z}+\frac{Q}{\rho c_{p}}\left(T-T_{0}\right)+\frac{D k_{T}}{c_{s} c_{p}} C_{z z} \\
& C_{t}+u C_{x}+v C_{y}+w C_{z}=D C_{z z}+\frac{D k_{T}}{T_{m}} T_{z z}
\end{aligned}
$$

where $u, v, w$ are respectively the components of velocity in $x$, $y, z$ directions, $t$ is the time, $T$ is the temperature, $C$ is the concentration species of the fluid, $v$ is the kinematic viscosity, $k_{0}$ is the material fluid parameter, $D$ is the coefficient of diffusion, $\alpha$ is the thermal diffusivity, $Q$ is the internal heat absorption/generation, $T_{m}$ is mean temperature, $\rho$ is the density of the fluid, $K_{T}$ is the thermal diffusion, $c_{s}$ is the susceptibility of concentration and $c_{p}$ is the specific heat.

The boundary conditions are

$$
\begin{aligned}
& z=0: u=u_{w}(x)=\frac{a x}{1-t \alpha}, v=v_{w}(y)=\frac{b y}{1-t \alpha}, w=0, T=T_{w}, C=C_{W} \\
& z \rightarrow \infty: u \rightarrow 0, v \rightarrow 0, u_{z} \rightarrow 0, v_{z} \rightarrow 0, C \rightarrow C_{\infty}, T \rightarrow T_{\infty}
\end{aligned}
$$

Here $a, b$ are positive constants. The similarity variables are used as given below.

$$
\begin{aligned}
& \eta=\sqrt{\frac{a}{v(1-\alpha t)}} z, u=\frac{a x}{1-\alpha t} f^{\prime}(\eta), v=\frac{a y}{1-\alpha t} g^{\prime}(\eta), \\
& w=-\sqrt{\frac{a v}{1-\alpha t}}(f(\eta)+g(\eta)), \theta=\frac{T-T_{\infty}}{T_{W}-T_{\infty}}, \phi=\frac{C-C_{\infty}}{C_{W}-C_{\infty}}
\end{aligned}
$$

Then the governing equations (1)-(5) become,

$$
\begin{aligned}
& f^{\prime \prime \prime}-f^{\prime 2}-\zeta\left(f^{\prime}+\frac{\eta}{2} f^{\prime \prime}\right)+f^{\prime \prime}(g+f) \\
& +K\left[-\zeta\left(2 f^{\prime \prime \prime}+\frac{\eta}{2} f^{i v}\right)+(g+f) f^{i v}+\left(f^{\prime \prime}-g^{\prime \prime}\right) f^{\prime \prime}-2\left(f^{\prime}+g^{\prime}\right) f^{\prime \prime \prime}\right]=0 \\
& g^{\prime \prime \prime}-g^{\prime 2}-\zeta\left(g^{\prime}+\frac{\eta}{2} g^{\prime \prime}\right)+(g+f) g^{\prime \prime} \\
& +K\left[-\zeta\left(2 g^{\prime \prime \prime}+\frac{\eta}{2} g^{i v}\right)+(g+f) g^{i v}+\left(f^{\prime \prime}-g^{\prime \prime}\right) g^{\prime \prime}-2\left(f^{\prime}+g^{\prime}\right) g^{\prime \prime \prime}\right]=0 \\
& \theta^{\prime \prime}+\operatorname{Pr}(g+f) \theta^{\prime}-\operatorname{Pr} \frac{\zeta}{2} \eta \theta^{\prime}+\operatorname{Pr} S \theta+\operatorname{Pr} D_{f} \phi^{\prime \prime}=0, \\
& \phi^{\prime \prime}+S c(g+f) \phi^{\prime}-S c \frac{\zeta}{2} \eta \phi^{\prime}+\operatorname{ScS} \operatorname{Sr} \theta^{\prime \prime}=0,
\end{aligned}
$$

With

$$
\begin{aligned}
& f(0)=0, f^{\prime}(0)=c, g(0)=0, g^{\prime}(0)=1, \phi(0)=1, \theta(0)=1, \\
& f^{\prime}(\infty)=0, g^{\prime}(\infty)=0, g^{\prime \prime}(\infty)=0, f^{\prime \prime}(\infty)=0, \phi(\infty)=0, \theta(\infty)=0,
\end{aligned}
$$

Where

$$
K=k_{0} a / v(1-\alpha t) \text { - Dimensionless viscoelastic parameter, }
$$

$\zeta=\alpha / a$ - Unsteady parameter,

$c=b / a-$ Stretching ratio,

$S c=v / D$ - Schmidt number,

$S r=D\left(T_{w}-T_{\infty}\right) K_{T} / T_{m}\left(C_{w}-C_{\infty}\right) T_{m} v$-Soret number,

$D f=D K_{T}\left(C_{w}-C_{\infty}\right) / c_{s} c_{p}\left(T_{w}-T_{\infty}\right) v$ - Dufour number,

$\operatorname{Pr}=v / \alpha$ - Prandtl number and

$\mathrm{S}$ - Heat absorption/generation parameter.

One can notice that the two-dimensional case $(g=0)$ is retained when $c=0$. For $c=1$, we can find an axisymmetric case $(f=g)$. The localized Nusselt and localized Sherwood numbers are represented by

$$
\begin{aligned}
& N u=\frac{x q_{w}}{k\left(T_{w}-T_{\infty}\right)}, \\
& S h=\frac{x j_{w}}{D\left(C_{w}-C_{\infty}\right)},
\end{aligned}
$$

With

$$
q_{w}=-\left.k \frac{\partial T}{\partial z}\right|_{z=0}, \quad j_{w}=-\left.D \frac{\partial C}{\partial z}\right|_{z=0},
$$

where $q_{w}$ denotes heat flux and $j_{w}$ denotes mass flux. Then, the dimensionless expressions of Nusselt and Sherwood numbers are

$N u \operatorname{Re}^{-\frac{1}{2}}=-\theta^{\prime}(0)$,

$\operatorname{Sh} \operatorname{Re}^{-\frac{1}{2}}=-\phi^{\prime}(0)$,

Where $\operatorname{Re}=u_{w} x / v$ is the local Reynolds Number?

\section{Homotopy Analysis Method}

The system of equations with boundary conditions is solved by Homotopy analysis method. We can choose the starting guesses as follows

$$
\begin{aligned}
& f_{0}(\eta)=-e^{-\eta}+1, g_{0}(\eta)=c\left(-e^{-\eta}+1\right) \\
& \theta_{0}(\eta)=e^{-\eta}, \phi_{0}(\eta)=e^{-\eta}
\end{aligned}
$$

Besides, we choose the auxiliary linear operators as

$$
\begin{aligned}
& L_{f}=\frac{\partial^{3} f}{\partial \eta^{3}}-\frac{\partial f}{\partial \eta}, L_{g}=\frac{\partial^{3} g}{\partial \eta^{3}}-\frac{\partial g}{\partial \eta}, \\
& L_{\theta}=\frac{\partial^{2} f}{\partial \eta^{2}}-\theta, L_{\phi}=\frac{\partial^{2} f}{\partial \eta^{2}}-\phi,
\end{aligned}
$$

which has the following properties,

$L_{f}\left(m_{1}+e^{\eta} m_{2}+e^{-\eta} m_{3}\right)=0, L_{g}\left(m_{4}+e^{\eta} m_{5}+e^{-\eta} m_{6}\right)=0$,

$$
L_{\theta}\left(e^{\eta} m_{7}+e^{-\eta} m_{8}\right)=0, L_{\phi}\left(e^{\eta} m_{9}+e^{-\eta} m_{10}\right)=0
$$

Where $m_{1}, m_{2}, m_{3}, m_{4}, m_{5}, m_{6}, m_{7}, m_{8}, m_{9}$ and $m_{10}$ are integral constants.

The series solution depends upon the non-zero parameters $h_{f}, h_{g}, h_{\phi}$ and $h_{\theta}$. In order to see the range of $h_{f}, h_{g}, h_{\phi}$ and 
$h_{\theta}$ which depend on $f^{\prime \prime}(0), g^{\prime \prime}(0), \phi^{\prime}(0)$ and $\theta^{\prime}(0)$, the H-curves of $f, g, \phi$ and $\theta$ are plotted. Figure 1(a) points out that the admissible limits of $h_{f}$ and $h_{g}$ are $-1.3 \leq h_{f} \leq-0.5$ and $-1.5 \leq h_{g} \leq-0.6$ respectively. Figure 1(b) represents that the limits of $h_{\phi}$ and $h_{\theta}$ are $-1.1 \leq h_{\phi} \leq-0.5$ and $-1.0 \leq h_{\theta} \leq-0.5$. We get a better accuracy in our results if we select the values of auxiliary parameter $h$ from this range. It is observed from the Figure 1 that $h_{f}=h_{g}=h_{\phi}=h_{\theta}=-0.9$ gives the better solution.

\section{Results and Discussion}

The governing nonlinear equations for the mass and heat transfer of a viscoelastic liquid over a stretched surface in the existence of internal heat generation/absorption, chemical reaction and radiation under the effect of Dufour-Soret parameters are solved by HAM. The influence of viscoelastic term $\mathrm{K}$ on the velocity components are depicted in Figure 2 (a-b). One can observe from these graphs that the velocity components decline for the ascending values of K. Figure $3(a-b)$ portrait the trends of temperature and concentration upon the action of $\mathrm{K}$ and we see that a rise in $\mathrm{K}$ results in the rise of both profiles. Figure $4(a-b)$ illustrate the influence of Dufour parameter and the heat generation/absorption parameter upon temperature. We notice here that the temperature soars for rising values of both Df and S. The change in concentration field due to Soret parameter is exhibited in Figure 5. We notice that the concentration rises as $\mathrm{Sr}$ ascends. The change in velocity field due to the stretching ratio is given in Figure 6. This figure indicates that the velocity rises for ascending values of the stretching ratio.

\section{Conclusion}

The changes due to Dufour-Soret effects on 3D boundary layer flow mass and heat transfer of a viscoelastic liquid upon a stretched surface is probed under the existence of chemical reaction, radiation and heat absorption/generation. The solution corresponding to this flow is attained by the application of HAM. The inferences can be summed up as follows.

- $\quad$ Rise in viscoelastic parameter $(\mathrm{K})$ results in deceleration of velocity components, whereas we can observe a rise in concentration and temperature because of the increase in the strength of viscosity.

- Temperature soars due to the rise in both Dufour (Df) and heat source parameter (S).

- As Soret parameter $(\mathrm{Sr})$ takes higher values, there is a rise in concentration observed.

- Velocity rises rapidly as the stretching ratio (c) ascends.

\section{References}

[1] Sakiadis BC (1961), Boundary-layer behavior on continuous solid surfaces:I. Boundary layer equations for two dimensional and axisymmetric flow. American Institute of Chemical Engineers (AIChE) Journal 7 (1), 26-28.

[2] Crane LJ (1970), Flow past a stretching sheet. Z. Angew. Math Phys. 21, 645-647.

[3] Wang CY (1984), The three-dimensional flow due to a stretching flat surface. Phys. Fluids 27, 1915-1917.

[4] Aziz MAE \& Nabil T (2012), Homotopy analysis solution of hydromagnetic mixed convection flow past an exponentially stretching sheet with Hall current. Math. Prob. Eng., doi: 10.1155/454023.

[5] Eswaramoorthi S, Bhuvaneswari M, Sivasankaran S \& Rajan S (2015), Effect of radiation on MHD convective flow and heat transfer of a viscoelastic fluid over a stretching surface. Procedia Engineering 127, 916-923.
[6] Bhuvaneswari M, Sivasankaran S \& Kim YJ (2012), Lie group analysis of radiation natural convection flow over an inclined surface in a porous medium with internal heat generation. Journal of Porous Media 15(12), 1155-1164.

[7] Postelnicu A (2010), Heat and mass transfer by natural convection at a stagnation point in a porous medium considering Soret and Dufour effects. Heat Mass Transfer 46, 831-840.

[8] Rushi Kumar B \& Sivaraj R (2011), Radiation and Dufour effects on chemically reacting MHD mixed convective slip flow in an irregular channel. Elixir Thermal Engg. 39, 4675-4683.

[9] Karthikeyan S, Bhuvaneswari M, Rajan S \& Sivasankaran S (2016), Soret and Dufour effects on MHD mixed convection heat and mass transfer of a stagnation point flow towards a vertical plate in a porous medium with chemical reaction, radiation and heat generation. J. Appl. Fluid Mech. 9(3), 1447-1455.

[10] Liao S (2009), Notes on the homotopy analysis method: some definitions and theorems. Comm. Nonlinear Sci. Num. Simul. 14(4), 983-997.

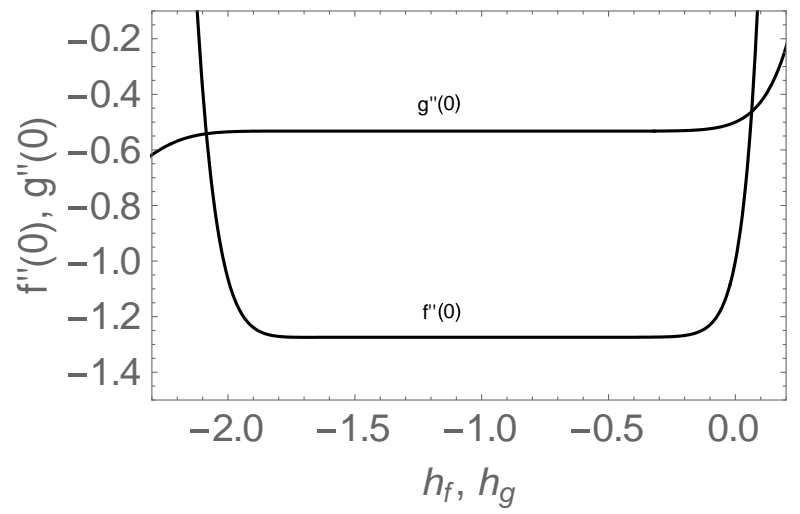

Fig. 1(a): h curves of $f^{\prime \prime}(0), g^{\prime \prime}(0)$.

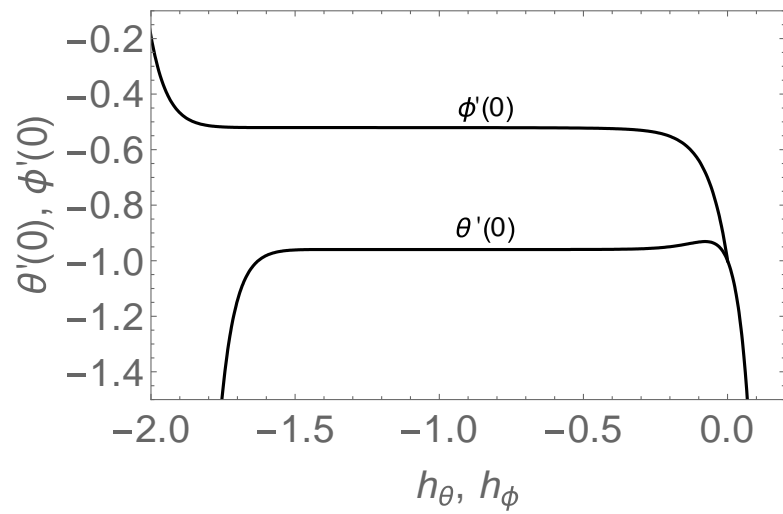

Fig. 1(b): h curves of $\theta^{\prime \prime}(0), \phi^{\prime \prime}(0)$.

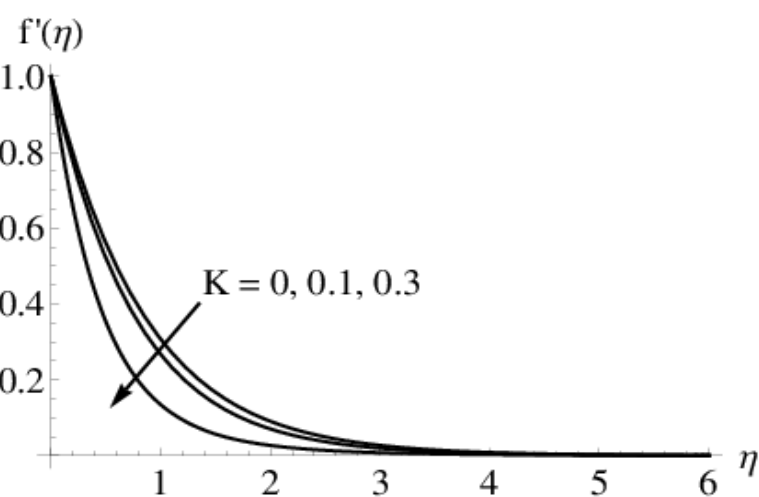

Fig. 2: Velocity profiles $f^{\prime}$ for different $K$ values 


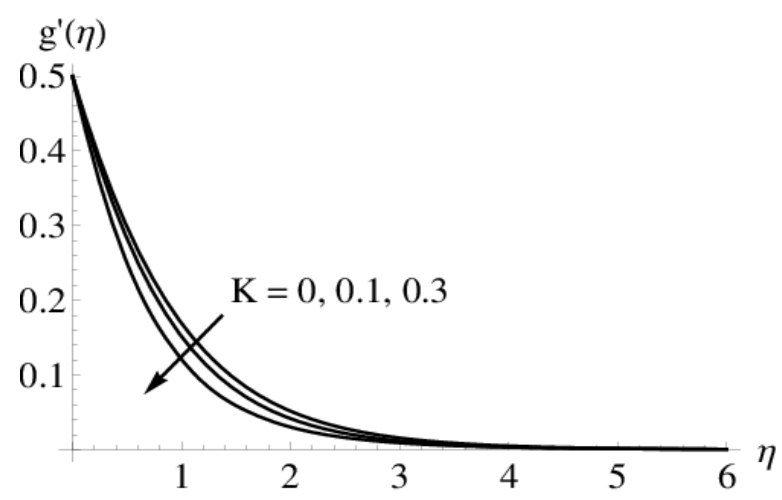

Fig. 3: Velocity profiles $g^{\prime}$ for different $K$ values

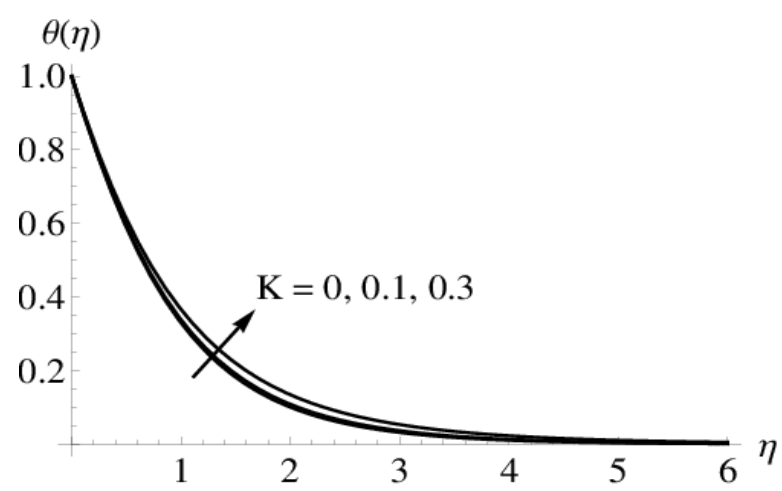

Fig. 4: Temperature profiles for dirrerent $K$ values

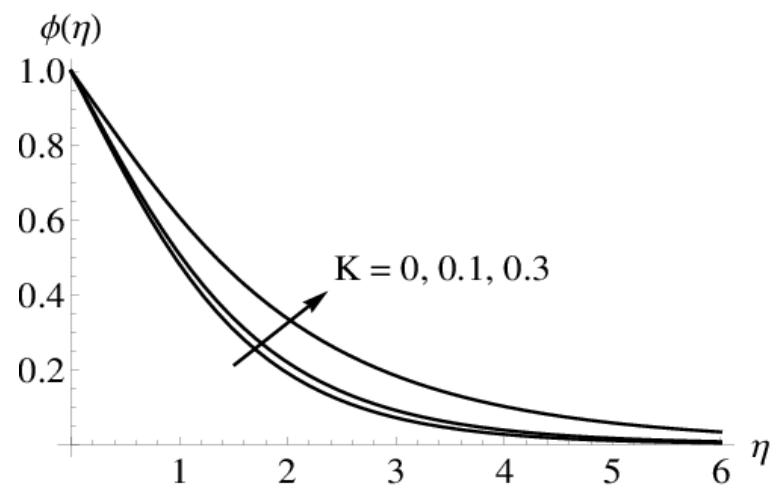

Fig. 5: Concentration profiles for dirrerent $K$ values

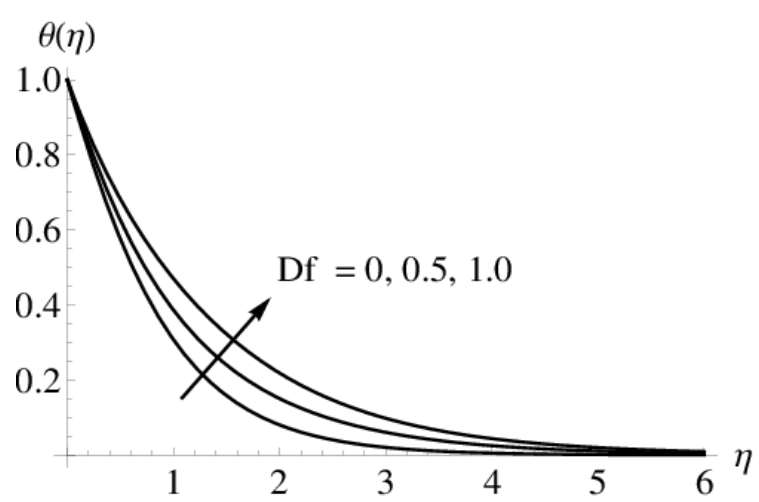

Fig. 6: Temperature profiles for dirrerent values of $D f$

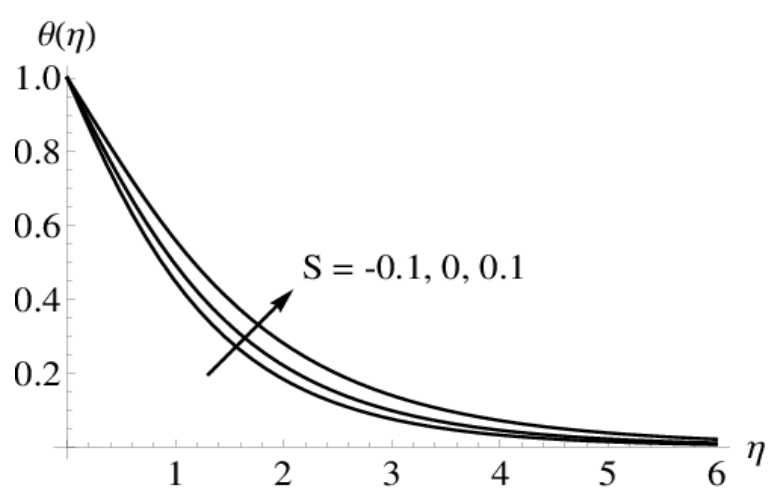

Fig. 7: Temperature profiles for dirrerent values of $S$

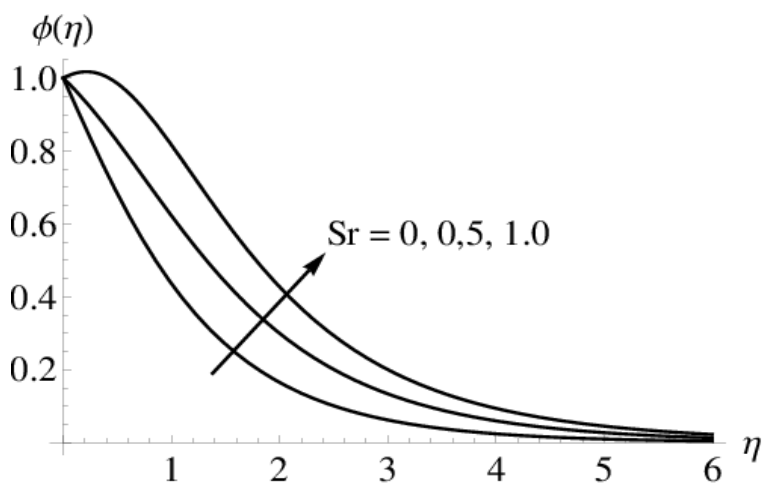

Fig. 8: Concentraction profiles for different values of Soret parameter

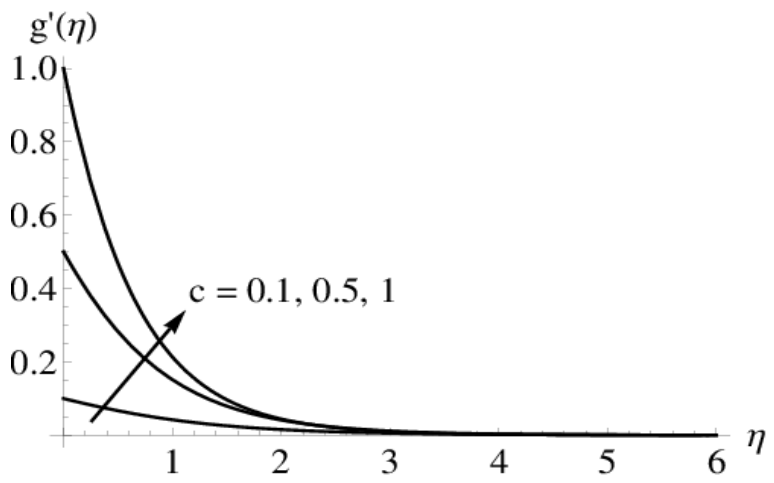

Fig. 9: Velocity profiles for different valures of $c$ 